\title{
Developing Globally Aware Engineers and Scientists in Nanotechnology
}

\section{Dr. Nancy Healy, Georgia Institute of Technology}

Nancy Healy is the Education and Outreach Coordinator for the National Nanotechnology Infrastructure Network (NNIN). NNIN is an NSF-funded user support network of 14 universities which also provides nano-education outreach activities and programs. Her office is located at Georgia Institute of Technology, Nanotechnology Research Center. Prior to joining the NNIN in 2004, she was a program manager at the S.C. Commission on Higher Education focused on science and math K-12 issues, teacher education, and teacher professional development. For ten years she served on the Board of Examiners for the National Council for the Accreditation of Teacher Education. She was also at the University of South Carolina for 17 years where she taught undergraduates, had an active research program in paleo-oceanography, and numerous graduate students. She has a B.S. in Zoology from the University of Rhode Island and an M.S. and Ph.D. in Geological Sciences from the University of South Carolina.

\section{Lynn Charles Rathbun, National Nanotechnology Infrastructure Network, Cornell University}

Dr. Lynn Rathbun is the Deputy Director of the National Nanotechnology Infrastructure Network and the Laboratory Manager of the Cornell Nanoscale Facility. As Deputy Director of NNIN, he is responsible for the management of all NNIN programs with particular emphasis on some of the various educational activities of NNIN. Dr. Rathbun received the B.S. degree in Physics from The Ohio State University in 1971 and the Ph.D. degree in Physics from the University of Illinois in 1979. He has been at Cornell University since 1979. 


\title{
Developing Globally Aware Scientists and Engineers in Nanoscale Science and Engineering
}

\begin{abstract}
The National Nanotechnology Infrastructure Network (NNIN) is a NSF-funded program which supports nanoscience researchers by providing state-of-the-art facilities and resources at 14 U.S. universities. (http://www.nnin.org). The NNIN has an education and outreach program with a mission to address the explosive growth of nanotechnology and its growing need for a skilled workforce. One of our goals is to encourage and develop talented students (undergraduate and graduate students) to become future leaders in nanoscale science and engineering (NSE). We have developed and implemented three programs that we hope will lead to globally aware scientists; these include the NNIN international Research Experience for Undergraduates program (iREU), the international Research Experience for Graduate students (iREG), and the international Winter Schools for Graduate Students (iWSG). This paper describes these three distinctly different programs and the outcomes we have determined through surveys of participants.
\end{abstract}

\section{Introduction}

Thomas Friedman's book The World is Flat, drew attention to the "flattening of the world" in the twenty-first century and how this "flattening" will greatly impact countries, societies, governments, and companies.[1] The publications Engineer of 2020 [2] and Educating the Engineer of 2020 [3] highlighted the importance of training globally competent engineers. The challenges are not only to train engineers who can work collaboratively with engineers around the world but also engineers who can solve the grand challenges facing the world today. These global linkages present new challenges as well as opportunities for educating engineering students. Much of the linkages that are and will occur are due to advances in technology with a major role being played by engineers. This in turn makes for new approaches for "doing" engineering, in particular acquiring a global perspective which includes an understanding of the different approaches to problem solving. Students should also develop knowledge about the complexities of the global environment and its social implications.

One study indicated that students' exposure to global perspectives should include how to "work effectively with people who define problems differently."[4] Full immersion in an international research environment should develop student understanding of these perspectives and bring these ideas back to the U.S. work environment. The U.S. economy is based on innovation and new technologies both of which have felt the effects of globalization. A recent report indicates that the U.S. must commit to embracing the global economy and train its workforce to be competitive in the global marketplace.[5] Many engineering programs are including global components in their curriculum to face this challenge. The National Academy of Engineering (NAE) report Infusing Real World Experiences into Engineering Education [6] includes in its best practices multidisciplinary team-based projects, cultural awareness, and problem-solving.

The importance of global education is recognized by the National Science Foundation (NSF) by its development of several programs focused on global placements. NSF stresses in its 
publications and programs the importance of supporting U.S. scientists and engineers to develop international collaborations that will help ensure that they gain international experience particularly early in their careers.[7] NSF has an entire division, Office of International Science and Engineering that supports the development of scientists and engineers who will become leaders in international collaborations that NSF sees as key to U.S. development and its role as a world leader in science and technology. The National Academy of Engineering also stresses the importance of a global perspective and in one study notes the recent major expansion of the STEM research workforce in the world.[5]\&[8] There has been a 35 percent increase in the U.S. and Europe research workforce and 50 percent in China. This increase has been attributed to the fostering of networks and collaborations across global boundaries through technology and is expected to have major implications for engineering in the future. The National Science Board has also recognized that one of the key challenges facing engineering is how do U.S. programs respond to the changing global context of engineering, technology and science. [9]

Providing a global experience in nanotechnology should be an important factor in developing a competent and highly skilled workforce. As noted in a recent National Academy of Engineering report [10] focused on the emerging U.S. manufacturing market. The report notes: "Manufacturing is changing in ways that may favor American ingenuity. Rapidly advancing technologies in areas such as biomanufacturing, robotics, smart sensors, cloudbased computing, and nanotechnology have transformed not only the factory floor but also the way products are invented and designed, putting a premium on continual innovation and highly skilled workers. .... Future manufacturing will involve a global supply web, but the United States has a potentially great advantage because of our tight connections among innovation, design, and manufacturing, and also our ability to integrate products and services." Clearly, training students in high technology fields such as nanotechnology while including a global perspective will help the U.S. keep its competitive edge. We believe that the experiences described herein will lead to the development of innovative and skilled scientists and engineers who can meet the challenges of new forms of manufacturing.

We report here on approaches to develop a global understanding of nanoscale science and engineering by immersing undergraduate and graduate students in research and/or courses in European countries, Japan, and developing nations. We have developed three international programs to provide undergraduates and graduate students experiences in a variety of global settings. These programs include variations and mixing of the four of the five typologies that can be used to achieve global competency by engineering students: international enrollment, international project, international work placement, and international field trip. [4]

\section{The National Nanotechnology Infrastructure Network - Education and Outreach Programs}

The National Nanotechnology Infrastructure Network (NNIN) is a NSF-funded program which supports nanoscience researchers by providing state-of-the-art facilities, support, and resources. (http://www.nnin.org) We also have a networked education program which has a variety of offerings for school-age children through adult professionals. Our mission is to address the explosive growth of nanotechnology and its growing need for a skilled workforce and informed public by offering education and training to individuals. We provide resources, programs, and materials to enhance an individual's knowledge of nanotechnology and its application to real-world issues. We believe that a strong U.S. economy requires a STEM- 
literate workforce ready to meet the technological challenges of a nano-enabled economy as well as an informed citizenry that supports continued and safe growth of nanotechnologies. One of our goals is to educate a dynamic workforce, one ready for the challenges of not only NSE but also the globalization of the world's economy. Towards this goal, we instituted three programs focused on international experiences for undergraduate and graduate students.

\section{International Research Experience for Undergraduates (iREU)}

The purpose of this program is to promote development of globally aware scientists by exposing promising young scientists and engineers, in this case talented undergraduates, to the promises and challenges of research in an international environment. We aim to demonstrate to these participants that not only is research in the global context a necessity in the 21st century, but it is also both exciting and well within their capabilities. By providing this opportunity early in their careers, we hope to have maximum long term impact. Towards this end, we have structured a program which selects the most promising candidates from a pool of already select students, have partnered with leading international nanotechnology research laboratories, and have provided the necessary support structure to make the experience challenging and rewarding. Our international partners include: National Institute for Materials Science (NIMS) in Tsukuba, Japan; the Forshungszentrum Julich in Germany; IMEC in Belgium; Delft University of Technology in The Netherlands; and Ecole Nationale Supérieure des Mines de Saint Etienne in France. NNIN established this program because we believe that globally aware scientists and engineers should be a priority in the $21^{\text {st }}$ century.

The NNIN iREU program builds upon the successful NNIN Research Experience for Undergraduates program (REU) which annually hosts approximately 80 students in an introductory nanotechnology research program at the 14 NNIN sites.[11] The NNIN REU has been in operation since 1997 and participants frequently asked if they could return for a second experience. In 2008, we developed the iREU to: allow participants a second nanotechnology research experience; encourage and support promising students in the STEM pipeline; and asssit in developing future STEM leaders with a global awareness. The NNIN REU program is used as a feeder and filter program for the iREU program, which is held in the " 2 nd summer", the summer after the initial REU experience. Only students who have successfully completed the prior summer NNIN REU program are eligible to apply for the next summer iREU program. This limitation assures that the students have a good level of basic laboratory expertise in NSE and can thus make the best of the international research opportunity offered to them.

Participants are selected using a variety of criteria: the application with several required written statements, his/her written REU project report, the video of the presentation at the REU convocation (end of summer meeting), $[\mathrm{x}]$ and the evaluations of the NNIN REU site coordinator, the REU project faculty member, and the REU project graduate student/post-doc mentor. Of these, the evaluation by the host faculty member is highly important in selecting those students who can best represent NNIN and who would most benefit from the program. Our ability to select only the most advanced and mature students is critical to the cooperation we receive from our international partners many who have not had undergraduate researchers in their facilities. The application site is a closed one that is only accessible to REU participants of a particular year, i.e, 2012 REU interns may access the application for 2013 iREU. 
We place approximately 16 students each summer at the international sites. The application allows the students to see the proposed projects at each international site along with the project director. Students indicate projects and sites that they are interested in from a list of proposed projects. Each selected student is paired with a project host or a senior staff scientist. Students are assigned to projects within their area of interest and consistent with their prior research experience. On a daily basis, the students generally interact with a postdoctoral researcher or graduate student, with regular significant interaction with the senior staff host. The students spend approximately 11 weeks at the host institution and become fully immersed in the research group and the research culture. Projects tend to be at a level higher than the prior summer's research project thereby building on their skill set. The placement settings not only have researchers from the host country but more often from several other countries which provides a truly international perspective to research ideas and approaches. Weekly check-in via the internet is required by all participants. The first report summarizes their project and follow-on reports detail the progress. This allows us to monitor the students and projects and address any issues as they occur.

Interns travel by group to the host site country with a member of the NNIN staff. The staff person stays until the interns are settled into the site (approximately 5-7 days). Prior to departure there is a one day meeting to cover such things as the facility they will be at, customs and language issues, etc. Students stay in either site housing or in apartments in the city the facility is located in.

This program provides an excellent career growth opportunity for the participants. iREU interns have indicated that their prior NNIN REU experience allowed them to meet the challenges of a more advanced project, work in a different research environment, and live and work with colleagues from other cultures. Of the 69 participants in the 5 years of this program, 45 are in graduate school; 12 are still undergraduates; 11 are working; and 2 are doing other. Several of those in graduate school have received NSF Graduate Fellowships, testament to the high quality of the participants and the boost that participation in this program offers.

We conduct two types of surveys of our participants - one immediately after the experience and another a year later to determine attitudes and impact further away from the experience. Table 1 summarizes the results of the iREU follow-up survey which occurs immediately after the summer program.

Table 1. Results of post-survey of iREU participants 2008-2012.

\begin{tabular}{|l|l|}
\hline Question iREU follow-up survey 2008-2012 n=51 & Avg. \\
\hline The research project was the right fit for my background and interests & 4.5 \\
\hline The project was an independent one with a strong intellectual focus & 4.3 \\
\hline I received quality technical support and meaningful scientific interactions & 4.6 \\
\hline The project increased my ability to work independently & 4.3 \\
\hline I devleoped improved technology skills in a specialised area of nanotechnology & 4.5 \\
\hline $\begin{array}{l}\text { The program provided a strong education experience inlcuding exposure to the } \\
\text { breadth of nanotechnology }\end{array}$ & 4.4 \\
\hline $\begin{array}{l}\text { The program provided information about different possible careers in } \\
\text { nanotechnology including those with an international component }\end{array}$ & 4.1 \\
\hline I learned to operate and communicate in an international research environment & 4.8 \\
\hline
\end{tabular}




\begin{tabular}{|l|l|}
\hline I developed relationships with international reserachers and colleagues & 4.8 \\
\hline $\begin{array}{l}\text { I developed an understanding of international research approaches and the global } \\
\text { nature of research }\end{array}$ & 4.8 \\
\hline $\begin{array}{l}\text { I consider my participation as a positive influence on my future educational or } \\
\text { career choices }\end{array}$ & 4.8 \\
\hline I developed a global perspective regarding research and society & 4.8 \\
\hline
\end{tabular}

Likert Scale 1-5 1 = poor/no 5= superior/very yes.

The results in Table 1 present some of the survey responses that we obtain from the participants soon after the completion of the summer program. We primarily want to know if their research experience was at an appropriate technical level and what their impressions of the global setting were. The results indicate that the projects are consistently at a high technical level and that the host sites provide sufficient technical support and scientific interactions. We have used these results to make adjustments to the programs and share any issues with our host sites. We consistently receive positive responses to the questions regarding their work in an international research environment and that the program has

The program exposed me to applied chemistry and materials science projects which were not available at my home institution, and consequently, I want to pursue these fields in graduate study. I developed my skills as an independent researcher and became more confident with my global research abilities. iREU 2012

This was a wonderful experience that helped me gain a strong understanding of the global variation in research practice as well as focus. iREU 2012

helped them to develop an understanding of the international approaches to research. While the students are working in laboratories in specific countries, the research groups at these facilities are multi-national. This allows the students to not only experience the culture of the host nation but also learn about the culture and research approach of individuals from other nations.

The iREU was a wonderful experience both culturally and research-wise. It has played such a large part in getting me where I am and the people I have met are connections I will always have. I really hope that the program can continue to give people the opportunity to work abroad and develop their research skills. iREU 2010

I really enjoyed learning about global research and being able to see how it's done in other countries. This experience gave me a deeper understanding of how boundaryless engineering really is. $i R E U 2011$
One of the primary goals of the iREU program is to have participants understand the global nature of science and engineering research. The follow-up survey results shown in Table 2 indicate that this goal has been attained as a high proportion of interns report that they feel confident and interested in continuing to work in an international setting. The program has been successful in helping the participants develop a global perspective regarding research and its impact on society. Importantly, nearly $2 / 3^{\text {rds }}$ of the respondents have maintained contact with their international colleagues. The quotes above from iREU interns are examples of how the program influenced them both professional and personally. We will continue to track these

participants as they advance in their education and career placements. 
Table 2. Results of follow-up survey of iREU participants 2008-2011.

\begin{tabular}{|l|l|}
\hline Question iREU follow-up survey 2008-2011 n=48 & Avg. \\
\hline $\begin{array}{l}\text { My iREU experience was important in securing my current position or } \\
\text { successfully performing my current position }\end{array}$ & 4.8 \\
\hline The program helped me feel confident to work in an international setting & 4.8 \\
\hline The program helped me to feel confident to work with international colleagues & 4.8 \\
\hline The program helped me to develop relationships with international researchers & 4.6 \\
\hline The program helped me to understand the global nature of science and engineering & 4.6 \\
\hline $\begin{array}{l}\text { The program helped me to develop an interest in working internationally (either as } \\
\text { a researcher or in a job) }\end{array}$ & 4.5 \\
\hline $\begin{array}{l}\text { I consider my participation to have appositive influence on my future educational } \\
\text { or career choice }\end{array}$ & 4.9 \\
\hline $\begin{array}{l}\text { The experience helped me to develop a global perspective regarding research and } \\
\text { society }\end{array}$ & 4.8 \\
\hline $\begin{array}{l}\text { I shared my knowledge about the international experience with colleagues at my } \\
\text { present position/current institution }\end{array}$ & 4.6 \\
\hline & $61 \%$ \\
\hline I have maintained contact with colleagues from the iREU site & $87 \%$ \\
\hline I have maintained contact with my NNIN iREU peers & $33 \%$ \\
\hline My NNIN iREU research resulted in an oral presentation & $15 \%$ \\
\hline My NNIN iREU research resulted in a journal article & $4 \%$ \\
\hline My NNIN iREU research resulted in a patent & \\
\hline
\end{tabular}

Likert Scale 1-5 $1=$ poor/no 5= superior/very yes.

\section{International Research Experience for Graduate Students (iREG)}

As an integral part of our relationship with NIMS Japan for hosting our iREU program participants, NNIN hosts a number of graduate students from Nanonet, the Japanese equivalent of NNIN, which is managed by NIMS. The goal of the iREG program is much the same as the iREU, that is, to increase awareness of the global nature of research.

Approximately 5 Nanonet graduate students (from institutions across Japan) come to the U.S. each summer to conduct research at NNIN facilities and faculty labs. NNIN solicits a group of projects from the NNIN sites suitable for graduate level research. Nanonet students complete an application and make a ranked selection of projects that they would like to collaborate on. NNIN management staff then matches students to projects. The projects provide enough variety of choices so that the participants are matched with research programs that complement and enhance their area of interest. Graduate students have ranged from those completing their first year to more advanced doctoral students. Nanonet covers the cost of travel, housing, and living expenses while NNIN provides the training and support to use the facilities.

Each of these students is at the NNIN site for 8-10 weeks during the summer at which time they are treated much like our REU students. In particular, they are integrated both socially and technically with the REU students, which adds greatly to their research and cultural experiences. Several of the iREGs have worked in the same lab as an REU intern fostering a strong international connection. Unlike undergraduate REU students, these graduate students come with a significant prior skill set and more focused scientific interests. During this time, they integrate into the appropriate faculty research group, are trained in equipment and techniques, and contribute to both their own research project and the overall goals of the research group. 
Since 2008, 18 students have been hosted at eight NNIN sites. While we do not have any survey data on these participants as they are part of the Japanese program, NIMS has indicated that they are extremely pleased with the interactions their students receive from the U.S. hosts. One graduate student returned to his host site for several more months and coauthored six papers with the host researcher. NNIN sites indicate that the REU interactions with the Japanese students provide an excellent, and often, first global connection with an international researcher. This also has inspired our REU students to apply for the iREU program for the following summer so that they may continue their development as global engineers and scientists.

\section{International Winter School for Graduate Students (iWSG)}

The international Winter School for Graduate Students (iWSG) is organized jointly by NNIN and institutions in developing countries with the goal of promoting international bridgebuilding and understanding by bringing together students and faculty in an intense teaching and societal experience. The objectives are to place nanoscience in the context of the developing world and demonstrate the societal and ethical dimensions of nanotechnology. In addition, we hope to establish relationships with foreign researchers while providing an intense course on select NSE topics.

Each year, approximately 10-15 U.S. graduate students and 5-7 U.S. faculty participate in a rigorous course in an emerging and research-intensive interdisciplinary NSE topic at a host institution in a developing country. Host country faculty also participate in the course offering. The technical course lasts six days and includes laboratory sections and quizzes. The technical course is followed by travel to a rural, underdeveloped part of the country ( 4 5 days) where students spend time observing, experiencing, and discussing societal challenges and the role science and technology can play in a developing society. A faculty representative from the societal/ethical area accompanies the graduate students into the field and fosters the discussion. A large group of students from the host country participate in the technical course and a smaller group joins in the rural experience. These two components of the course allow for interactions among both student groups. The winter school is a comprehensive education program whose content is archived at the NNIN education portal. See, e.g. http://www.nnin.org/nnin_grads.html for complete access to course materials.

U.S. students are selected from across the country and do not have to be from an NNIN site for consideration for participation. Approximately 45 applications are received annually from our call for participation to science and engineering departments across the country. Each applicant completes an online application which includes written responses and faculty recommendations. We seek students with strong academic backgrounds, an interest in societal issues related to technology, and who have the potential to become leaders in their field.

The iWSG program began in 2008 and we have completed four courses at the following sites:

- IIT Kanpur, India: Organic Electronics -12/2008

- IIT Mumbai, India: Nanoelectronics - 12/2009

- IISc Bangalore, India: Nanofabrication - 1/2011

- University at Campinas, Brazil: Silicon and III-V Nano Photonics Campinas, Brazil $1 / 2012$

U.S. students complete an evaluation instrument for each of the iWSGs and the results are too 
numerous to be presented in this paper. Table 3 provides an example of some of the results for iWSG 2009.

Table 3. iWSG 2009Survey Results $n=64$

\begin{tabular}{|l|l|}
\hline To what extent did this course: & Avg. \\
\hline Give you a broad perspective of the field and its challenges & 4.3 \\
\hline Introduce/discuss nanoscale MOSFET and current research & 4.4 \\
\hline Provide introductory theoretical understanding of high k and metal gates & 4.2 \\
\hline Introduce variability issues/sources for devices and SRAMs & 4.0 \\
\hline Provide understanding of major fabrication technologies & 4.1 \\
\hline Discuss issues of interconnections and reliability & 3.7 \\
\hline Provide an understanding of non-volatile memories & 3.9 \\
\hline Provide understanding of device transport \& nanoscale modeling & 4.1 \\
\hline Introduce circuit techniques for digital and analog & 3.7 \\
\hline Provide effective form to discuss critical technical issues & 3.9 \\
\hline Provide a systems constraint perspective to design & 4.0 \\
\hline $\begin{array}{l}\text { Allow you to interact across international boundaries and see the other } \\
\text { world perspective }\end{array}$ & 4.3 \\
\hline $\begin{array}{l}\text { How do you rate the course in its effectiveness as an approach to } \\
\text { teaching/learning and bridge building }\end{array}$ & 4.5 \\
\hline
\end{tabular}

Likert scale: $1=$ not at all; $2=$ not significantly; $3=$ somewhat; $4=$ significantly; $5=$ great extent

Overall, the courses receive very good ratings including providing a broad perspective to the targeted field and its challenges as well as allowing participants to interact across international boundaries and see other world perspectives. The latter was an important goal of the program in that we are seeking to develop globally aware scientist through this experience, a major focus of the program. Participants in the field trip portion of the trip complete an essay on their thoughts and observations. These essays indicate that students are extremely positive about the workshop and the field trip. The visits to rural villages in India were "eye-opening" events for all participants and helped them to see how technology connects and potentially can help the poorest people in the world. Sample comments by the US participants are below and these examples indicate the cultural impact of the global experience:

- "I didn't expect to see such a big diversity in the population here; Brazil seems to have drawn immigrants from many different countries."

- "I feel that the itinerary for the week gave an excellent overview and an in-depth look into education, technology, and life in general throughout India. There are huge challenges for development and modernization within India.

- "A Brazilian student shared his perspective that his interaction with our group has erased some of the prejudices he had about Americans. We agreed that prejudices on both sides were broken down by the two week experience."

- "While we've routinely interact with graduate students from developing nations (e.g. China, India, Turkey), we've noted that we rarely get to interact with Brazilian students. For that reason, the ability to network with so many Brazilian students (an 
opportunity we rarely get at conferences, even those that are international) was a transformative experience."

- "From the trip to the rural area in India, I learned that we, as engineers and scientists, must share the responsibility of increasing people's quality of life in rural areas. The reason is that we do have the infrastructure and knowledge needed to investigate solutions to daily problems (and people from villages do not..... The iWSG provided an excellent experience in understanding people's lives in rural areas and in making us aware of our role in the quality of life of society in general."

In fall 2011, we instituted a new survey to follow-up with participants to gain information on how they perceived the technical and societal portions of the course years to months after their participation. To date, 24 of the 33 participants (from the first 3 years) have completed the survey. This survey will become ongoing and will be administered annually several months ( 12 months) after participation in the iWSG. Results for the technical and societal pieces of the survey are presented in Table 4. The results demonstrate that the technical portion of the course does an effective job in presenting the technical aspects and that the topics have been at the forefront of new knowledge. The societal portion of the program received very high marks and clearly demonstrates that exposure to the underdeveloped world is extremely important in developing a global perspective in these young scientists and engineers. Ninety percent of the respondents indicated that the iWSG was time well spent in their academic career and 68\% indicated that the experience has had an impact on their views of technology and society.

Table 3. Follow-up survey results of iWSG participants 2008-2011

\begin{tabular}{|l|l|}
\hline Technical Portion Questions iWSG 2011* (participants from IWSG) & Avg. \\
\hline The course was the correct level for my background and experience & 3.6 \\
\hline The presenters were very knowledgeable and added to my understanding of & 4.5 \\
\hline The course provided the right balance of lecture, labs, and discussion & 3.4 \\
\hline The course provided the host country's perspective on the topic & 4.0 \\
\hline The course provided an effective forum to discuss critical technical issues & 3.3 \\
\hline The course duration was sufficient for the topics covered & 4.2 \\
\hline The course topic was timely and provided current and cutting-edge & 4.3 \\
\hline Societal Portion Questions iWSG 2011*(participants from IWSG 1,2,3) & Avg. \\
\hline It allowed me to identify/perceive the world context of technology & 4.7 \\
\hline $\begin{array}{l}\text { It allowed me to see how technology can help improve the lives of under- } \\
\text { served populations }\end{array}$ & 4.6 \\
\hline It allowed me to put my research in the context of the global arena & 4.1 \\
\hline It allowed me to have discussions with the foreign participants about & 4.8 \\
\hline It opened up my understanding of technology and the impact on society & 4.6 \\
\hline It has influenced my future in terms of my career choices & 4.1 \\
\hline Reflection questions post experience (1+ years since iWSG) & Avg. \\
\hline I believe that my iWSG experience was very worthwhile - time well spent & 4.6 \\
\hline The experience has had an impact on my technical direction & 3.5 \\
\hline The experience has had an impact on my views of technology and society & 4.7 \\
\hline The experience has made me think more about ethics in scientific research & 4.4 \\
\hline
\end{tabular}




\begin{tabular}{|l|l|}
\hline I have shared my iWSG experience with colleagues at my institution & 4.9 \\
\hline $\begin{array}{l}\text { The experience has made me think about my research with a global } \\
\text { perspective }\end{array}$ & 4.7 \\
\hline
\end{tabular}

*Likert scale $1-51$ = poor no 5 = superior/very yes

While the survey results shown in Table 3 represent self-reported data, they do provide insight into the quality of the technical program, the impact of the societal experience, and the impact the iWSG has had on participants several months after they took part in the course. Under the technical portion, the topics and level of materials were deemed very good but the students were not as positive about time for discussion. This was clearly stated in their written comments that more time should be allowed for discussion with their foreign participants. We have noted this for planning of future courses but also see this as a positive point in that the students were eager to learn more from their global counterparts - an important component of the program.

The societal portion of the course also received very high marks in allowing the students to place technology in the global arena and how technology can help improve the lives of underserved populations. While we did not expect the societal portion of the course to alter career choices, the results indicate that the participants now think of their research in a world context and that the experience may influence their future career choices. With time to reflect on the experience, participants indicated that even a year or two after the course it still influenced their views on technology and society. We believe that it is very important to expose graduate students to the impact of technology on the majority of the world's population. This is especially important in developing future leaders to meet the Grand Challenges of the National Academy of Engineering. [x]

\section{Conclusion}

The NNIN has developed three distinct programs to encourage the development of globally aware undergraduate and graduate students. We have been fortunate to have excellent foreign partners who fully support these programs and their continued development. For the iREU program, the students are place at facilities that typically support post-doctoral or graduate student level international researchers. Placement of undergraduate researchers in these facilities has been a new experience for some of the sites yet our hosts have been quite pleased with the ability of the students that have participated and in the amount they have been able to accomplish in a short period. Survey results indicate that the experiences of the undergraduates have achieved the goal of developing a global awareness of NSE research. The iREG program has provided a venue for Japanese students to experience the U.S. approach to research but also allows our U.S. hosts and students to gain a perspective on the Japanese culture. Success of this program is the continued support of Nanonet to fund its students' participation. Lastly, the iWSG has had a significant impact on U.S. participants especially with regards to the societal dimensions of the program. Creating such opportunities for both academic and cultural exchange of ideas will play an important role in the development of future STEM leaders. The NNIN international programs demonstrate that the participants benefit from the approaches used and have developed a global perspective that will be important in their career paths.

\section{Acknowledgements}

This work is supported by the National Nanotechnology Infrastructure Network under NSF ECS0335765 award and OISE-1030533. 


\section{References Cited}

[1] T. L. Friedman, The World is Flat 3.0:A Brief History of the Twenty-First Century, Farrar, Straus and Giroux, New York, 2007.

[2] National Academy of Engineering, Engineer of 2020: Visions of Engineering in the New Century, National Academy Press, Washington, DC, 2004.

[3] National Academy of Engineering, Educating the Engineer of 2020, National Academy Press, Washington, DC, 2005.

[4] G.E. Downey, J.C., Lucena, B.M. Moskal, R. Parkhurst, T. Bigley, C. hays, B.K. Jesiek, L. Kelly, J. Miller, S. Ruff, J.L. Lehr, A. Nichols-Belo, ”The Globally Competent Engineer: Working Effectively with People who Define Problems Differently", Journal of Engineering Ed. Vol. 95, No. 2, pp.107-122.

[5] National Academy of Engineering, Global Technology: Changes and Implications, National Academy Press, Washington, DC, 2011.

[6] National Academy of Engineering, Infusing Real World Experiences into Engineering Education, National Academy Press, Washington, DC, 2012.

[7] Office of International Science and Engineering, National Science Foundation, Accessed at: http://www.nsf.gov/div/index.jsp?div=OISE

[8] National Academies of Science Engineering and Medicine, Rising Above the Gathering Storm, National Academy Press, Washington, DC, 2007.

[9] National Science Board, Moving Forward to Improve Engineering Education, National Science Foundation, Arlington, VA, November 2006.

[10] National Academy of Engineering, Making Value: Integrating Manufacturing, Design, and Innovation to Thrive in the Changing Global Economy, National Academy Press, Washington, DC, 2012.

[11] National Nanotechnology Infrastructure Network, "REU Program", Accessed at: http://www.nnin.org/nnin reu.html. 